\title{
Caracterización de unidades de producción de camote (Ipomoea batata) en San Luis, Cañete
}

\author{
Characterization of sweet potato production units \\ (Ipomoea batata) in San Luis, Cañete
}

Norma Carolina Valverde ${ }^{1 *}$, Raúl Blas Seminario ${ }^{1}$, Rember Pinedo Taco ${ }^{1}$

\section{RESUMEN}

El cultivo de camote en el valle de Cañete es de alta importancia económica y alimenticia y por la superficie sembrada es el principal abastecedor de esta raíz que ingresa al mercado mayorista de Lima. El objetivo del estudio fue tipificar y caracterizar los sistemas de producción de camote en el distrito de San Luis, Cañete, Lima. Con un enfoque multidimensional, se aplicaron encuestas con preguntas estructuradas a una muestra representativa de 50 productores cuya principal actividad económica se sustenta en la producción de camote. Mediante técnicas estadísticas de análisis multivariado se definieron los componentes y conglomerados. Con el Análisis de Componente Principal (ACP) se determinaron cinco nuevas variables sintéticas que explicaron el 74\% de la varianza, mientras que con el análisis de conglomerados se hallaron pequeños y medianos productores (no se encontraron grandes productores). El 82\% de los sistemas de producción practican el monocultivo y sólo el $6 \%$ agricultura diversificada hasta con tres cultivos por parcela. Los pequeños productores siembran en promedio 3,56 ha, con un rendimiento promedio de $20548 \mathrm{~kg} / \mathrm{ha}$, lo que les genera un ingreso neto mensual de $2078 \mathrm{PEN}$. En cambio, los medianos productores de $6,11 \mathrm{ha}$, con un rendimiento medio de $21780 \mathrm{~kg} / \mathrm{ha}$, perciben 5811 PEN como ingreso neto mensual.

Palabras clave: agricultura familiar, conglomerado, monocultivo, rentabilidad, tipología de productor.

\begin{abstract}
Sweet potato cultivation in the Cañete Valley is of high economic, and food importance and for the cultivated area, it is the chief supplier of this root that enters the wholesale market of Lima. The objective of the study was to typify and characterize sweet potato production systems in the district of San Luis, Cañete, Lima. With a multidimensional approach, surveys with structured questions were applied to a representative sample of 50 producers whose main economic activity is based on sweet potato production, and using statistical techniques of multivariate analysis, new synthetic and conglomerate variables were defined. With the Principal component analysis (PCA), five new synthetic variables were defined that explained $74 \%$ of the variance; while with the cluster analysis small and medium producers were found, (no large producers were found). The $82 \%$ of the production systems practice monoculture systems and only $6 \%$ diversified agriculture with up to three crops per plot. Small producers cultivate an average of $3.56 \mathrm{ha}$, with an average yield of $20,548 \mathrm{~kg} / \mathrm{ha}$, which generates a net monthly income of $2078 \mathrm{PEN}$; while the medium producers of $6.11 \mathrm{ha}$, with an average yield of 21,780 kg/ha with 5811 PEN as monthly net income.
\end{abstract}

Keywords: conglomerate, family farming, monoculture producer typology, profitability.

\section{Introducción}

En el Perú la geografía agropecuaria es heterogénea, con una multiplicidad de climas y pisos altitudinales utilizados para la agricultura, separados entre sí por otras clases de tierras (desierto costeño, selva tropical o zonas de alta montaña) donde se distribuyen distintos sistemas de producción (Maletta,
2017). A nivel de costa existe una estrecha franja irrigada por ríos que discurren por cuencas desde las partes altas de las cordilleras del lado occidental y gracias a la disponibilidad de agua los valles ubicados en la costa han desarrollado sistemas de producción con mediana y gran tecnología. Uno de ellos es el valle de Cañete en el departamento de Lima, zona agrícola con más de 20 mil hectáreas.

\footnotetext{
1 Universidad Nacional Agraria La Molina (UNALM). Lima, Perú.

* Autor para correspondencia: 20150520@lamolina.edu.pe
} 
Según los resultados del IV Censo Nacional del Agro-2012 (IV CENAGRO, 2012), en el Perú existen 2 millones 260 mil 973 productores, de los cuales el $90 \%$ son considerados pequeños y medianos productores y solo el $10 \%$ se encuentra en el grupo de grandes productores con más de 100 ha (INEI, 2013). Con respecto a las unidades agropecuarias (UAP), a nivel nacional se cuenta con 2 millones 213 mil 506, lo que representa un promedio de 17,5 hectáreas de tierra por unidad agropecuaria (INEI, 2013). En el ámbito de estudio que comprende el departamento de Lima (valle de Cañete), las mismas fuentes indican que hay 69022 ha con una superficie promedio de 7,2 ha por UAP.

Respecto a la tenencia de tierras a nivel nacional, el 79,6\% de productores poseen parcelas con un tamaño menor a las 5 hectáreas, mientras que el $9.9 \%$ desde 5 ha hasta 9.9 ha. De estos, el $5.3 \%$ tiene de 10 a 19.9 ha y solo el $0.10 \%$ más de 100 ha (INEI, 2013). Según Maletta (2017), Pinedo et al. (2017) e IICA (2015), el primer grupo correspondería a agricultores de minifundio, el segundo a pequeños productores y el tercero a grandes productores.

Por otro lado, los sistemas productivos no están formados por explotaciones homogéneas (Maletta, 2017; Coronel de Renolfi y Ortuño, 2005; Rodríguez, 2002), ya que existe una gran diversidad de estas, con diferentes características físicas, socioeconómicas y técnicas (Pinedo et al., 2017, 2018), por lo que se requiere conocerlas y analizarlas (Escobar y Berdegué). El conocimiento de los sistemas y la tipología de productor son herramientas importantes y necesarias para la adecuada planificación o propuesta de políticas de fomento (Borja et al., 2016; Coronel de Renolfi y Ortuño, 2005; Rocha et al., 2016). Mediante la tipificación se puede agrupar a productores con características de manejo, producción y técnicas similares, tomando en cuenta que algunos están ubicados en zonas geográficas delimitadas (Escobar y Berdegué, 1990; Escobal y Armas, 2015).

Respecto a los métodos de tipificación de productores, algunos plantean definirlos por sus sistemas de producción como orgánicos, tradicionales, mixtos y convencionales (Pinedo et al., 2018), mientras que otros proponen por sistemas de agricultura familiar: de subsistencia, de transición y agricultura familiar consolidada
(CAN, 2011). Lo concreto es que no se encontrarán explotaciones homogéneas ya que existe una gran diversidad (Coronel de Renolfi y Ortuño, 2005).

Desde el punto de vista metodológico, también existe ambigüedad conceptual para definir adecuadamente las variables representativas para una medición válida de rasgos que caracterizarían a un tipo de productor (Maletta, 2017). Pese a estas dificultades, hay evidencias de tipificación tradicional como pequeños, medianos y grandes productores (Coronel de Renolfi y Ortuño, 2005; Pinedo et al., 2017). Sin embargo, existe consenso en que las innumerables variables que se utilizan para tipificar las explotaciones agrícolas actúan conjuntamente, y no por su forma unitaria (Coronel de Renolfi y Ortuño, 2005; Pinedo et al.). De manera simple la tipificación de productores puede basarse en estudios realizados previamente y en la información con que cuentan los entes territoriales, buscando un primer acercamiento con los lugares objeto de estudio (Rocha et al., 2016).

En función de estos resultados se requiere establecer criterios para tipificar las fincas. El INEI (2013) ha establecido tipologías de productor con base en la variable tenencia de tierras. Algunos estudios plantean el uso de variables como el tamaño de parcela, tipo de explotación, nivel de tecnologías y relaciones con los mercados (Maletta, 2017). Otro criterio para tipificarlas considera las características individuales de los agricultores como edad, sexo, educación; de sus unidades agropecuarias (tierra, ganado, mano de obra familiar y contratada) y del contexto en el que operan como altitud, acceso a bienes y servicios públicos, distancia respecto a los mercados de productos (Pinedo et al., 2017).

En términos sociales, se considera minifundistas a todos aquellos productores cuyas unidades agropecuarias tienen un tamaño menor a 5 hectáreas, y en el Perú representan el 89\% del total de agricultores. El mediano productor representa el $10 \%$ y el gran productor el $1 \%$ (Vásquez, 2016). Maletta (2017) afirma que a nivel nacional, 2,13 millones son unidades clasificables convencionalmente como pequeña agricultura familiar (PAF), mientras que 61.000 UAP son clasificables como ajenas a la pequeña agricultura familiar (no-PAF) por su tamaño, por el empleo de trabajo asalariado o porque el productor es una persona jurídica. 
Por lo expuesto, la investigación se enfoca en determinar los tipos de productores más representativos de la zona, elaborar modelos que grafiquen el desempeño de los sistemas, identificar factores limitantes y recursos potencialmente productivos en el área, y plantear alternativas técnicas que contribuyan a mejorar el desempeño de las unidades de producción de camote en el valle de Cañete.

\section{Materiales y métodos}

La investigación se realizó en el distrito de San Luis, ubicado en el valle de Cañete, a 104 msnm en la zona sur de la región Lima, localizada entre los paralelos $12^{\circ} 16^{\prime} 02^{\prime \prime}$ y los $13^{\circ} 04^{\prime} 36^{\prime \prime}$ " de latitud sur y entre los meridianos $78^{\circ} 56^{\prime} 00^{\prime \prime}$ y $76^{\circ} 23^{\prime} 04^{\prime \prime}$ de longitud oeste. Presenta una precipitación promedio anual de $26.6 \mathrm{~mm}$. La investigación fue descriptiva teniendo en cuenta la unidad central de análisis: Unidad Agrícola de Producción (UAP): parcela de camote (Maletta, 2011; Cáceres \& Julca, 2018), así como el medio donde se desarrolló. Comprende la revisión de información secundaria y la identificación de información faltante respecto a la situación de la producción del cultivo de camote, sus condiciones actuales y su prospectiva en el escenario nacional (Pinedo et al., 2018).

Durante el desarrollo de la investigación se consideró la participación de los productores y otros actores relevantes de la cadena productiva del camote a nivel distrital, además de obtener información real sobre la función que cumple cada componente en la zona de estudio (Rocha et al., 2016; Pinedo et al., 2018). Luego de determinar la población finita se obtuvieron 50 productores de camote mediante una muestra irrestricta aleatoria (Scheaffer et al. 1987). La información de cada una de las parcelas se logró a través de la aplicación de encuestas con un cuestionario de preguntas estructuradas (Coronel de Renolfi y Ortuño, 2005; Hurtado y Hurtado, 2015; Rocha et al., 2016) relacionadas con aspectos técnicos, económicos, sociales y ambientales (Pinedo et al., 2018; Cáceres y Julca, 2018). La unidad objeto de análisis fue, por lo tanto, la propiedad rural, identificada aquí como una Unidad Agrícola de Producción (UAP) y definida como una unidad privada de organización de la producción, mediana o grande, desde aquellas consideradas de minifundio con menos de una hectárea hasta más de 20 catalogadas como pequeños productores (Maletta, 2017; Pinedo et al., 2018).

\section{La tipificación de sistemas productivos}

Las UAP fueron analizadas como sistemas productivos en los cuales actúan, simultáneamente, innumerables variables estructurales, sociales, económicas y ecológicas (Coronel de Ronolfi y Ortuño, 2005). Se empleó el análisis estadístico multivariante, para resumir y clasificar datos provenientes de poblaciones en las cuales se han medido dos o más características (Bravo et al., 2016). Por tanto, se utilizaron las técnicas de análisis de componentes principales (ACP) y análisis clúster simplificación estructural, y la de clasificación o agrupación (Coronel de Ronolfi y Ortuño, 2005). Con estas técnicas se logró ordenar, resumir y clasificar datos provenientes de poblaciones en las cuales se han medido dos o más características. Con el análisis clúster se conformaron grupos que facilitaron la clasificación y análisis (Escobar y Berdegué, 1990; ENEI, 2012). Asimismo, se identificaron aquellas variables que contribuían a la clasificación de los predios, eliminando información redundante (Rocha et al., 2016).

\section{Procedimiento estadístico}

Primero, se realizó la correlación de las variables, además de sus estadísticos descriptivos y de frecuencia a fin de estandarizarlas. Se aplicó la prueba de Kaiser, Meyer y Olkin (KMO) y la de esfericidad de Bartlett para definir el grado de estandarización (Borja et al., 2016; Ruiz et al., 2012). Luego se usó el método de Análisis de Componentes Principales (ACP) para obtener las comunalidades y la prueba de varianza total que contribuyó a registrar el número de componentes mínimos que permitieron elaborar la matriz de componentes y la prueba de rotación con el método de Normalización Varimax. Después se realizó el análisis de conglomerados con la vinculación de Ward, y al final la tabla que resume los conjuntos en variables sintéticas. La primera prueba fue para la reducción de la información e identificación de las variables que más explicaron los conjuntos agrupados; y el segundo para un agrupamiento eficiente de los datos, que al final derivó en la 
clasificación de los sistemas e identificó sus principales diferencias. Los análisis se realizaron con SPSS versión 21.

\section{Resultados y discusión}

El valle de Cañete es una zona privilegiada para el desarrollo de la agricultura debido a la calidad de sus tierras y condiciones climáticas. El cultivo de camote requiere condiciones mínimas como temperatura, humedad y horas sol (IICA, 2016; Flórez et al., 2016). Los productores en estudio se dedican a ese cultivo con la finalidad de mejorar sus ingresos económicos, acceso a bienes y servicios, pero posiblemente con escasa responsabilidad ambiental por los sistemas intensificados de producción agrícola. Por lo tanto, el manejo sostenible de la producción de camote en términos económicos, ambientales y sociales no sería factible (Sarandón y Flores, 2014).

\section{Aspectos socioculturales de las UAP}

Con respecto a la responsabilidad en el manejo de las UAP, el $86 \%$ está a cargo de hombres, mientras que el $14 \%$ de mujeres. Este aspecto puede ser desventajoso, debido a que las mujeres han demostrado a través de la historia que tienen mayor responsabilidad ambiental y en la conservación de recursos como la variabilidad de semillas (Maletta, 2011; Pinedo et al., 2018). La edad de los productores puede estar asociada a los años de experiencia en el cultivo de camote y a las posibilidades de adopción de tecnologías. El 52\% de los agricultores se encuentran en el rango de 32 a 69 años, que correspondería al de mejor productividad; el 36\%, entre 70 y 79 años, son adultos mayores; y el $12 \%$ ancianos mayores a
80 años (Figura 1A). Esto puede deberse a que la población joven menor a 32 años se encuentra en las ciudades o centros poblados realizando otras actividades no necesariamente agrícolas. El 54\% de los productores tienen educación secundaria y solo el $18 \%$ educación técnica y universitaria (Figura 1B).

La edad del productor puede influir en la adopción de nuevas tecnologías de producción o su inserción al mercado. Sin embargo, un factor que puede jugar un rol importante es el nivel de educación (CYNMIT, 2003). De acuerdo al estudio realizado, el $98 \%$ cuenta con algún nivel de educación: el 54\% de los productores tienen educación secundaria y el 18\% educación técnica y universitaria (Figura 1B). Estos resultados coinciden con los del INEI (2018), según los cuales, el 50,4 $\%$ cuenta con educación secundaria y $20,7 \%$ con educación superior.

Con respecto al lugar de residencia, el 94\% de los productores viven en un centro poblado próximo a sus UAP, lo que les permite seguir de cerca sus actividades productivas. Solo el $2 \%$ vive en la ciudad y el 4\% en la chacra. Según Ayora (2015), Pinedo et al. (2018), en la zona rural la situación es inversa por la tendencia a la migración hacia las ciudades, por lo que están alejados de sus UAP. Otro factor que puede diferenciar las tipologías de producción (Escobar y Berdegué, 1990; Pinedo et al., 2017), es que el 58\% tiene la condición de propietarios de tierras agrícolas y $42 \%$ de arrendatarios, que por lo general son agricultores migrantes.

En el estudio se encontraron productores que manejan menos de 3 ha, menos de 5 ha, hasta 10 ha y hasta 21 ha. Con estos valores es posible establecer tipologías de productores (Figura 2). Al respecto, Maletta (2017) indica que existe una alta
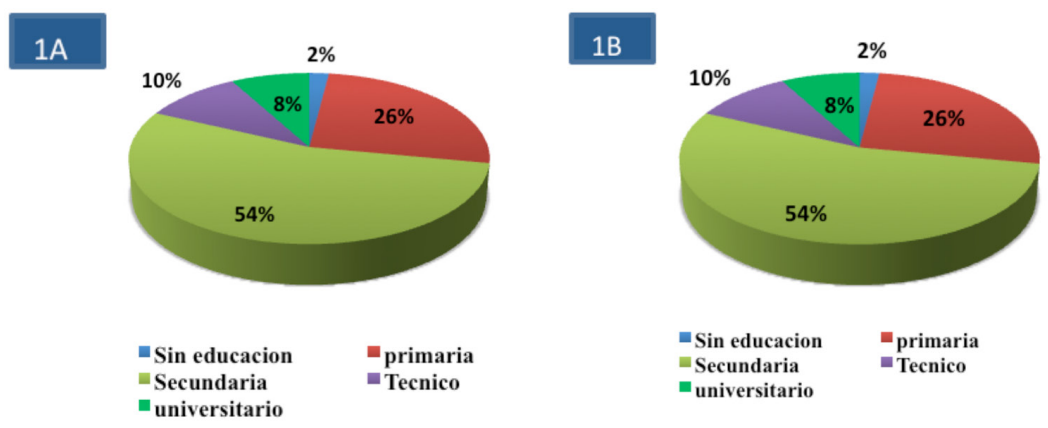

Figura 1. Edad y nivel de educación de los productores de camote en San Luis-Cañete, 2018. 


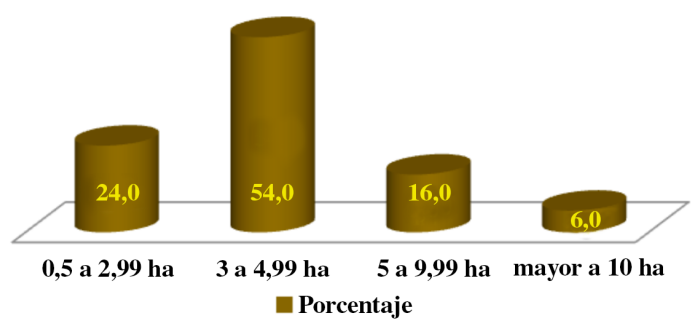

Figura 2. Clasificación de tenencia de tierras (ha) de UAP de camote en San Luis-Cañete, 2018.

atomización de tierras en el Perú, especialmente en la sierra. El INEI (2013) destaca que en la costa peruana, de acuerdo a la superficie cultivada que manejan, existen pequeños, medianos y grandes productores.

En términos generales se observa un alto nivel de satisfacción de los productores de camote con esta actividad, puesto que les genera ingresos económicos y seguridad alimentaria. El $82 \%$ y $10 \%$ están muy contentos y contentos, mientras que el $6 \%$ y $2 \%$ muestran poca satisfacción o no están satisfechos. El cultivo de camote, aparte de su importancia económica por su alto contenido de betacarotenos sobre todo en variedades de pulpa amarilla, cumple un rol estratégico en la seguridad alimentaria local y nacional (Flórez et al., 2016).

El 68\% de los productores contratan mano de obra para sus actividades agrícolas de la producción de camote, mientras que el $20 \%$ de la fuerza de trabajo corresponde a familiares y contratados. Por lo tanto, se puede afirmar que la tipología de productor predominante es agricultura no-familiar (Maletta, 2017).

El sistema de producción predominante en la zona es el monocultivo. El 70\% de la actividad agrícola se basa en sistemas de monocultivo, mientras que un $22 \%$ siembra dos cultivos pero con bajo nivel de asociación y el $8 \%$ maneja hasta tres cultivos asociados. Las prácticas de monocultivo en el corto plazo pueden ser viables económicamente para los agricultores, pero la diversificación productiva puede conducir hacia sistemas de producción más sostenibles (Pinedo et al., 2018; Sarandón y Flores, 2014). En el valle de Cañete se siembran 72 cultivos, entre frutas, raíces y tubérculos (Plan Desarrollo Concertado de la Provincia Cañete 2008-2021).

El gasto medio por productor en fertilizantes es intermedio. Los productores aplican entre 10 y 15 sacos de fertilizantes. Los niveles de fertilización generalmente recomendados para la costa peruana son relativamente bajos de N-P-K 80-60-120 (Villagómez, 2017) e intermedios 110120-180 (Molina, 2004). Entre las principales plagas que afectan al cultivo de camote en las UAP estudiadas se reportan insectos plaga y un tipo de virus (SPFMV). Se registraron niveles de daño alto (3), medio (2) y bajo (1), como se observa en la Tabla 1.

\section{Análisis de componentes principales (ACP)}

En el análisis de estadísticos descriptivos y la prueba de ji-cuadrada Pearson las variables seleccionadas son independientes y las más apropiadas para explicar los conjuntos que se agruparon (Tabla 2). La medida de adecuación muestral de Kaiser-Meyer-Olkin (KMO) con 0,503 indica que las variables se encuentran correlacionadas a nivel aceptable y son útiles para explicar las características de las UAP. La prueba de esfericidad de Bartlett y el valor aproximado de la ji-cuadrada $(\mathrm{p}=0.001)$ confirman tal afirmación (Velásquez y Peresgrovas, 2017). Para el ACP se consideraron 17 variables originales cuyo $\mathrm{CV}$ era igual o mayor a $40 \%$, que midieron aspectos productivos y socioeconómicos de los productores y sus UAP.

Tabla 1. Principales plagas en el cultivo de camote en San Luis-Cañete.

\begin{tabular}{llc}
\hline Nombre vulgar & \multicolumn{1}{c}{ Nombre científico } & Nivel daño \\
\hline Gusano ejército & Spodoptera frugiperda & 3 \\
Mosca blanca & Bemisia tabaci & 3 \\
Chacarero & Anómala sp. & 2 \\
Gorgojo & Euscepes postfasciatus & 2 \\
Comedor hoja & Spodoptera eridania & 2 \\
Moteado plumoso & SPFMV & 1 \\
Cigarrita & Empoasca sp & 2 \\
\hline
\end{tabular}

Tabla 2. Eigenvalues y total de varianza explicada.

\begin{tabular}{cccc}
\hline \multirow{2}{*}{ Componente } & \multicolumn{3}{c}{ Autovalores iniciales } \\
\cline { 2 - 4 } & Total & \% de varianza & \% acumulado \\
\hline 1 & 4,049 & 23,815 & 23,815 \\
2 & 3,134 & 18,433 & 42,248 \\
3 & 2,277 & 13,394 & 55,642 \\
4 & 1,461 & 8,597 & 64,239 \\
5 & 1,147 & 6,748 & 70,986 \\
\hline
\end{tabular}


El procedimiento de reducción de factores permitió visualizar el comportamiento de las variables y determinó cuatro factores en los que se pueden agrupar en cuatro nuevas variables sintéticas. De 64 variables, la técnica de ACP ha permitido reducir a 17 que explican el 70,986\% de la varianza.

En la Tabla 3 se muestran los vectores propios de la matriz de correlaciones de los cinco componentes principales. El CP1 explica el 28,5\% de la varianza total, por lo tanto, es el componente principal más influyente en el análisis y el que mejor expresa las diferencias entre las UAP y las características de los sistemas productivos de camote (Coronel de Ronolfi y Ortuño, 2005). Las variables VAR1, VAR2, VAR3 Y VAR4 fueron las más influyentes. Dentro de este componente se observa la importancia de la disponibilidad de jornales en las tareas agrícolas, aplicaciones de agroquímicos y su relación directa con la posesión de maquinaria agrícola, por lo que se les denomina "labores agrícolas".

En el segundo componente (CP2), las variables más influyentes fueron VAR5, VAR6, VAR7 y VAR8, que explican el 18,33\% de la varianza. El CP2 refleja la sostenibilidad económica de las familias que cultivan camote en sus UAP y, por lo tanto, se le considera como "ingreso por UAP".

El CP3 explica el 13,39\% de la varianza y entre las variables que lo representan se encuentran
VAR9, VAR10, VAR11, VAR12. Está vinculado a la edad del agricultor, su situación en cuanto a la posesión de tierras, sus costos de producción y la productividad de las parcelas. En las encuestas se ha observado que el $35 \%$ de los agricultores tienen la condición de arrendatarios, por lo tanto, es una situación crítica para el manejo sostenible del cultivo, ya que no pueden hacer ninguna mejora $\mathrm{y}$, por el contrario, realizan actividades extractivas con alto uso de productos químicos, lo cual puede ser muy costoso.

El CP4 está representado por las variables VAR13, VAR14 y VAR15, que explican el 8,60\% de la varianza. Muestra la relación que existe entre la cantidad de materia orgánica, agua de riego y dependencia de insumos externos, por lo que se le denominó "dependencia de insumos externos". El modelo de producción predominante es el monocultivo con alto uso de insumos externos.

Finalmente el CP5, que explica el 6,75\% de la varianza, presentó correlación elevada con las variables VAR16 y VAR17. Está conformado por las variables calidad de tierras y nivel de integración social, por lo que se le denominó "nivel organizativo y recursos". Los suelos de costa son sometidos a labranza intensiva con escasas prácticas conservacionistas, y, por lo tanto, los productores se ven obligados a rotar de lugar de siembra en busca de parcelas con tierras de mejor calidad, lo

Tabla 3. Matriz de componentes rotados con Eigenvectores de la matriz de correlación para los cinco componentes principales de mayor relevancia.

\begin{tabular}{llrrrrr}
\hline & \multicolumn{1}{c}{ Variables } & \multicolumn{5}{c}{ Componente } \\
\hline Clave & \multicolumn{1}{c}{ Nombre } & \multicolumn{1}{c}{ CP1 } & \multicolumn{1}{c}{ CP2 } & \multicolumn{1}{c}{ CP3 } & CP4 & CP5 \\
\hline VAR1 & $\mathrm{N}^{\text {o } \text { aplicaciones }}$ & .908 & .011 & -.072 & .196 & -.022 \\
VAR2 & Costo plaguicidas & .897 & .003 & -.103 & .203 & -.056 \\
VAR3 & Posesión maquinaria & .674 & .158 & -.037 & -.151 & -.117 \\
VAR4 & $\mathrm{N}^{\circ}$ jornales & .606 & .105 & .344 & .291 & .322 \\
VAR5 & Ingreso neto mensual & -.014 & .912 & -.302 & .032 & -.104 \\
VAR6 & Costo beneficio & .197 & .799 & .316 & -.043 & -.135 \\
VAR7 & Superficie siembra ha & -.139 & .717 & -.442 & -.034 & -.050 \\
VAR8 & Precio por kg & .313 & .679 & .154 & -.121 & -.128 \\
VAR9 & Edad del agricultor & -.018 & .158 & .814 & .158 & .016 \\
VAR10 & Costo por hectárea & -.041 & .147 & -.701 & .342 & -.205 \\
VAR11 & Tenencia tierras & -.169 & -.119 & .569 & .041 & .257 \\
VAR12 & Rendimiento kg/ha & -.123 & .460 & -.549 & .447 & -.206 \\
VAR13 & Pago agua de riego & .118 & -.061 & -.102 & .839 & -.119 \\
VAR14 & Cantidad materia orgánica & .107 & -.073 & .049 & .806 & .066 \\
VAR15 & Dependencia insumos externos & .416 & .026 & .136 & .517 & -.509 \\
VAR16 & Calidad tierras & -.052 & -.083 & .191 & -.087 & .807 \\
VAR17 & Nivel integración social & .022 & -.222 & .200 & .007 & .731 \\
\hline
\end{tabular}


cual es un factor que puede debilitar las escasas formas asociativas y trabajos colectivos.

\section{Análisis de los conglomerados de las UAP de camote en Cañete}

Mediante el análisis de conglomerados se establecieron dos clústeres, con una línea de corte en el nivel 18 (Figura 3). Dos grupos es un número razonable considerando que las subdivisiones subsiguientes del árbol darían origen a grupos menos diferentes entre sí. Asimismo, podría resultar poco práctico para los agentes de cambio trabajar con más de cinco tipos diferentes de finca.

Al realizar el análisis clúster resultan dos CG que tienen semejanzas entre los productores de cada grupo, pero difieren de uno a otro grupo (INEI, 2002). El conglomerado 1 (CG1) está conformado por pequeños productores porque la superficie de cultivo de camote en promedio es 3,42 ha (Tabla 4). Al respecto, Pinedo et al. (2017) y Maletta (2017), así como el INEI (2013) mencionan que la superficie de cultivo es una variable adecuada para la tipificación de productores además de otras variables socioeconómicas y que las superficies
Tabla 4. Características de los clúster 1 y 2 en agroecosistemas de San Luis-Cañete.

\begin{tabular}{lcc}
\hline \multicolumn{1}{c}{ Variables } & Clúster 1 & Clúster 2 \\
\hline Edad (años) & 59 & 56 \\
Superficie siembra (ha) & 3,42 & 6,11 \\
Variedades (N ${ }^{\circ}$ de variedades) & 2 & 3 \\
Fertilizantes (kg) & 485 & 642 \\
Gasto fertilizantes (PEN) & 970 & 1284 \\
Gasto en plaguicidas (PEN) & 431 & 522 \\
Jornales (Jornales/ha) & 60 & 66 \\
Rendimiento (kg/ha) & 20083 & 21789 \\
Costo de producción por ha & 6206 & 6532 \\
Ingreso neto mensual (PEN) & 1847 & 5812 \\
Relación B/C & 1,3 & 1,9 \\
\hline
\end{tabular}

de tierras cultivables menores a cinco hectáreas se consideran como pequeños productores.

La productividad del cultivo de camote en promedio es $20.083 \mathrm{~kg} / \mathrm{ha}$ con un costo de $6206 \mathrm{PEN}$.

Las semejanzas y diferencias entre los dos clústeres establecidos se observan en la Tabla 1. Con respecto a la edad de los productores, ambos grupos no difieren significativamente, pero en

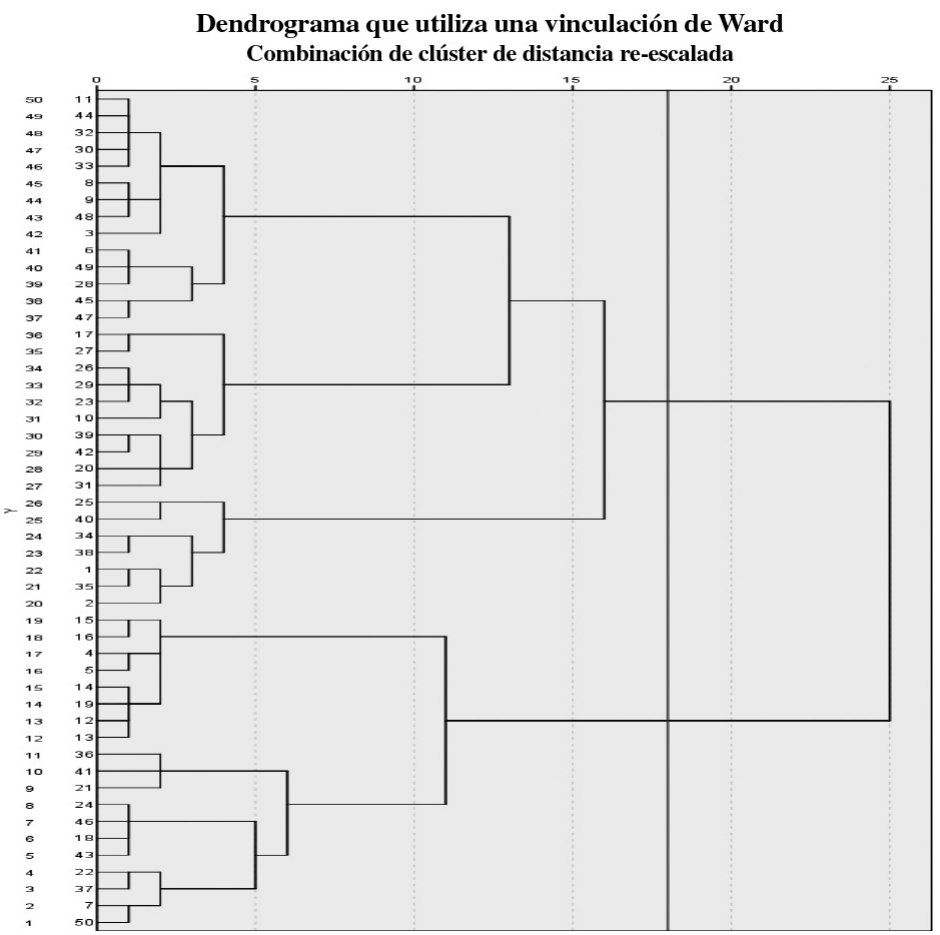

Figura 3. Agrupamiento de pequeños y medianos productores de camote en San Luis, Cañete. 
superficie sembrada, en el clúster 1 la media es 3,42 ha por productor, mientras que en el clúster 2 la media es 6,11 ha. La media de rendimiento es mayor en el clúster 2 que llega hasta $21.789 \mathrm{~kg} / \mathrm{ha}$.

El INM de los productores del clúster 1 es 1847 PEN con una relación B/C de 1.3, mientras que en el clúster 2 el INM es 5812 PEN con una relación $\mathrm{B} / \mathrm{C}$ de 1.9. Esto significa que los del grupo 2, por cada sol invertido logran una rentabilidad de 1,9 PEN y generan un ingreso neto mensual de 1847 PEN, resultando la relación $\mathrm{B} / \mathrm{C}$ igual a 1,3 . Por cada PEN invertido obtienen 0.30 PEN de beneficio.

El conglomerado 2 (CG2) se caracteriza porque las UAP son mayores que el CG1 y califican como medianos productores con un promedio de 6,11 ha. El costo de producción por hectárea alcanza hasta 6532 PEN, lo cual les genera un INM de 5812 PEN, con una relación $\mathrm{B} / \mathrm{C}$ de 1,90 PEN.

Entre los factores que influyen en los altos rendimientos alcanzados en ambos conglomerados están las condiciones climáticas y la experiencia de los agricultores en el manejo del cultivo. Al evaluar las características agronómicas de 61 clones en la localidad de Cañete, Reynoso (2003) halló un rendimiento promedio de $23.5 \mathrm{t} / \mathrm{ha}$; mientras que con las variedades comerciales Huambachero y Jhonatan la media alcanzó rendimientos de 10,6 y 27,3 t/ha, respectivamente. Según Flórez et al. (2016), las condiciones idóneas para su cultivo son una temperatura media durante el período de crecimiento, superior a los $21^{\circ} \mathrm{C}$, y un ambiente húmedo (80-85\%). Tal condición la cumple el valle de Cañete. El rendimiento promedio del cultivo de batata en el mundo es de 12,60 t/ha, cifra que depende de la variedad que se siembre.

Los datos obtenidos han hecho posible identificar tipos de sistemas de producción en los cuales, por ejemplo, el efecto de las variables agroecológicas fue menos determinante que el de variables socioeconómicas, o viceversa (Escobar y Berdegué, 1990). La aplicación de los métodos multivariados entregó una ponderaci6n objetiva de la influencia de cada variable sobre la determinación del sistema de las UAP (Borja et al., 2016).

Bajo el enfoque de sistema de finca, al analizar la influencia de variables referidas a los objetivos y habilidades de los productores en el manejo de sus UAP y aquellas que se deben a fenómenos socioeconómicos, agroecológicos, tecnológicos e institucionales, se observó que no implicó la asignación de un peso igual ni constante a cada una de las variables, en relación con cada posible tipología de producción (Escobar y Berdegué, 1990; Maletta, 2017; Pinedo et al., 2018).

En cuanto a las técnicas estadísticas utilizadas, el ACP expresó la relación entre múltiples variables cuantitativas, transformando las variables originales en otro conjunto de variables sintéticas denominadas componentes.

\section{Conclusiones}

Se encontraron dos tipos de productores. El primero corresponde al grupo de pequeños productores que manejan hasta 3.42 ha de cultivo de camote, mientras que el segundo corresponde al de medianos productores que manejan en promedio 6.11 ha pudiendo llegar hasta 21 ha.

El monocultivo es la práctica que predomina en la zona de estudio. El 70\% de los productores practican cultivos en limpio, mientras que el $28 \%$ mantiene prácticas tradicionales con dos cultivos diversificados.

Solo el $20 \%$ de los productores recurren a la mano de obra familiar y contratados para las labores agrícolas. El 68\% contrata mano de obra.

La producción de camote resulta rentable en términos económicos. El valor de la relación B/C es positivo para ambos grupos de productores.

\section{Literatura Citada}

Ayora, L.

2015. Caracterización geoespacial de la cuenca media y baja del río Supe para identificar sistemas agrícolas prevalentes. Aporte Santiaguino, 2(3): 39-65.

Borja, M.; Reyes, L.; García, J.A.; Almeraya, S.X.

2016. Tipología de productores de uva (Vitis vinífera L.) en Aguascalientes, México. Revista Mexicana de Ciencias Agrícolas, 7 (2): 249-261.
Cáceres Yparraguirre, H.; Julca Otiniano, A.

2018. Caracterización y tipología de fincas productoras de vid para pisco en la región Ica-Perú. Idesia (Arica), 36(3): 35-43.

CAN.

2011. Agricultura Familiar Agroecológica Campesina en la Comunidad Andina. Comunidad andinas de NacionesSecretaria General. 


\section{CIMMYT}

1993. La adopción de tecnologías agrícolas: Guía para el diseño de encuestas. Programa de Economía del CIMMYT. Centro Internacional del Maíz y Trigo. México, D.F., México. 85 p.

Coronel de Renolfi, M.; Ortuño, S.

2005. Tipificación de los sistemas productivos agropecuarios en el área de riego de Santiago del Estero, Argentina. Problemas del Desarrollo. Revista Latinoamericano de Economía, 36 (140): 63-88.

Coronel de Renolfi, M.; Ortuño, S.

2005. Tipificación de los sistemas productivos agropecuarios en el área de riego de Santiago del Estero, Argentina. Problemas del Desarrollo. Revista Latinoamericano de Economía, 36 (140): 63-88.

Escobar, G.; Berdegué, J.

1990. Tipificación de sistemas de producción agrícola. Red Internacional de Metodología de Investigación de Sistemas de Producción. Santiago, Chile. 269 p.

Escobal, J.; Armas, C.

2015. El uso de encuestas y censos agropecuarios para desarrollar una tipologia de la pequeña y mediana agricultura familiar en el Perú. En: Escobal, J.; Fort, R., Zegarra, E.; Escobal, J.; Fort, R.; Zegarra, E (Eds.) Agricultura peruana: nuevas miradas desde el Censo Agropecuario. GRADE. Lima, Perú.

Flórez, C.H.; Uribe, C.P.; Contreras, C.A.

2016. Mosquera. Perspectivas tecnológicas y comerciales para el cultivo de la batata en Colombia. Corporación Colombiana de Investigación Agropecuaria (Corpoica). Colombia. 110 p.

Hurtado, A.L.; Hurtado, C.L.

2015. La toma de decisiones en investigación educativa IICA. con SPSS. Primera edición. 376 p.

2015. Estudio: Mercado y producción de quinua en el Perú. Instinto Interamericano de Cooperación para la INEI. Agricultura. Lima, Perú. 175 p.

2018. Indicadores de educación por departamentos, 20082018. Instituto Nacional de Estadística e Informática. Lima, Perú. 339 p.

INEI.

2002. Guía para la aplicación del análisis multivariado a las encuestas de hogares. Dirección Técnica de Demografía e Indicadores Sociales. Instituto Nacional de Estadística e Informática. Lima, Perú. 63 p.
2013. Resultados definitivos. IV Censo Nacional Agropecuario 2012. Instituto Nacional de Estadística e Informática. Lima, Perú. 62 p.

Maletta, $\mathrm{H}$.

2017. La pequeña agricultura familiar en el Perú. Una tipología microrregionalizada. En IV Censo Nacional Agropecuario 2012: Investigaciones para la toma de decisiones en políticas públicas. Libro V. FAO. Lima, Perú. 176 p.

Molina, J.P.

2004. Manejo del cultivo del camote para mercado interno y exportación. INIEA. Lima, Perú. 16 p.

Pinedo, R.; Gómez, L.; Julca, O.

2017. Caracterización de sistemas de producción de quinua (Chenopodium quinoa Wiilld) en el distrito de Chiara, Ayacucho. Aporte santiaguino, 10 (2): 351-364.

Pinedo, R.; Gómez, L.; Julca, O.

2018. Sostenibilidad de sistemas de producción de quinua (Chenopodium quinoa Wiilld).Ecosistemas y Recursos Agropecuarios, 5 (15): 399-409.

Reynoso, D.

2003. Desarrollo de productos de camote para América Latina: Materia seca de las raíces de camote. Identificación de variedades para procesamiento. Informe Técnico final del Proyecto FONTAGRO. CIP - Perú. 98 p.

Rocha, C.; Mora, J.; Romero, J.C.

2016. Tipología de sistemas de producción en la zona rural del municipio de Ibagué, Colombia. Agronomía Mesoamericana, 27 (2): 253-264.

Sarandón, S.J.; Flores, C.

2014. Agroecología: bases teóricas para el diseño y manejo de agroecosistemas sustentables. Argentina. 466 p.

Scheaffer, R.; Mendenhall, W.; Ott, L.

1987. Elementary Survey Sampling. 5th edition. Duxbury Press, Belmont Publishing. Boston, US. 464 p.

Vásquez V.

2016. Agricultura peruana: promesas, desconocimiento y olvido. Primera edición. 127 p.

Velázquez-Avendaño, J.A.; Perezgrovas-Garza, R.

2017. Caracterización de sistemas productivos de ganado bovino en la región indígena XIV Tulijá-Tseltal-Chol, Chiapas, México. Agrociencia, 51(3): 285-297.

Villagómez, V.

2017. El cultivo de camote. Programa de Raíces y Tuberosas. Universidad Nacional Agraria La Molina. Perú. 44 p. 
